Revista Brasileira de Tecnologias Sociais | ARTigos | DOI: 10.14210/RBTS.v5N1.PO2-09

\title{
ACOLHIMENTO NA ESTRATÉGIA SAÚDE DA FAMÍLIA: UMA PROPOSTA DE REORGANIZAÇÃO DO ACESSO
}

\section{WELCOMING IN THE FAMILY HEALTH STRATEGY: A PROPOSAL TO REORGANIZE ACCESS}

\author{
Eliane Cristine Lyra Oliveira Viana ${ }^{1}$ \\ Rita de Cássia Gabrielli Souza Lima ${ }^{2}$
}

RESUMO: $\mathrm{O}$ artigo relata a experiência de implantação do acolhimento em uma equipe de Saúde da Família (ESF) de uma Unidade Básica de Saúde(UBS) de um município do Sul do Brasil. Os procedimentos introduzidos pelo planejamento foram derivados de encontros coletivos em reuniões de equipe e com usuários. São eles: extinção de fichas de porta; pacto de responsabilidades; sensibilização; construção de um fluxograma de atendimento; e criação de uma agenda integrada. A implantação da proposta redimensionou o processo de trabalho da equipe e qualificou o acesso ao cuidado. Conclui-se que a grandeza do acolhimento está exatamente na simplicidade de sua complexidade.

PALAVRAS-CHAVE: Acolhimento; Atenção básica; Sistema Único de Saúde.

ABSTRACT: This article describes the experience of implementing the welcoming system of a Family Health Team (ESF) at a Primary Health Center (UBS) in a municipality in the south of Brazil. The procedures introduced by the planning team were decided upon as a result of collective meetings of the team with users. They comprise: elimination of entrance tickets; agreement on responsibilities; raising awareness; the creation of an attendance flowchart; and, the creation of an integrated agenda. The proposal implementation re-dimensioned the team working process and qualified the care access. The conclusion drawn is that the greatness of welcoming is exactly in the simplicity of its complexity.

KEYWORDS: Welcoming; Primary Care: Unique Health System.

Licença CC BY:

Artigo distribuído sob os termos Creative Commons, permite uso e distribuição irrestrita em qualquer meio desde que o autor credite a fonte original.
1 Mestranda em Saúde e Gestão do Trabalho da Universidade do Vale do Itajaí UNIVALI. Enfermeira da Estratégia da Saúde da Família da Prefeitura Municipal de Itajaí (SC). E-mail: eclyra@hotmail.com.

2 Doutora em Saúde Coletiva pela Universidade Federal de Santa Catarina - UFSC. Docente e pesquisadora da Escola de Ciências da Saúde da Universidade do Vale do Itajaí - UNIVALI. E-mail: rita.lima@univali.br. 


\section{INTRODUÇÃO}

Este artigo relata a experiência de implantação do acolhimento em uma equipe de Saúde da Família (ESF) de uma Unidade Básica de Saúde (UBS) de um município do Sul do Brasil.

$\mathrm{O}$ acolhimento pode ser definido como uma ferramenta de trabalho utilizada por equipes de saúde na relação com o usuário do serviço de saúde. Trata-se de uma proposta de organização do processo de trabalho por meio de uma escuta qualificada. Corresponde a uma das diretrizes da Política Nacional de Humanização (PNH) e também da Política Nacional de Atenção Básica (BRASIL, 2010a).

A imagem-objetivo do acolhimento é a qualificação dos serviços do Sistema Único de Saúde (SUS). O acolhimento não é um espaço ou um local, mas uma ação (dimensão política) precedida de exercício reflexivo (dimensão ética), na medida em que se aplica em realidades que envolvem terceiros com necessidades de saúde, e/ou tratamento de doenças e/ou ações específicas (LYRA et al., 2016). Não pressupõe, portanto, hora especial ou profissional específica para realizá-lo. Ele implica compartilhamento de saberes e fazeres, devendo ser compreendido como um processo desenvolvido no encontro, entre indivíduo/família/comunidade e trabalhadores de saúde, de modo integral e resolutivo (BRASIL, 2011). É uma diretriz ético-política (BRASIL,2010a) de humanização das diferentes instâncias do SUS (CARMAGNANI, 2005), apresentada aos serviços pelo Ministério da Saúde (MS) em 2004 (BRASIL, 2010b).

A Política Nacional de A tenção Básica (PNAB) tem como uma de suas diretrizes "possibilitar o acesso universal e contínuo a serviços de saúde de qualidade e resolutivos”, abordando dentro dessa perspectiva que os serviços de Atenção Básica devem se organizar para assumir a função central de acolher, escutar e oferecer uma resposta positiva, sendo capaz de resolver a maioria dos problemas de saúde da população. A proximidade e a capacidade de acolhimento, vinculação, responsabilização e resolutividade são fundamentais para a efetivação da Atenção Básica como contato e porta de entrada preferencial da rede de atenção (BRASIL, 2011, p. 3).

Nesse sentido, entende-se que acolhimento é um modo de operar e reorganizar os processos de trabalho da ESF, com disposição para a escuta da narrativa e para o esforço de resolução da necessidade ou encaminhamento para um nível ascendente de complexidade (BRASIL, 2010a).

Segundo Maeyama (2007), os serviços de saúde têm se mostrado pouco capazes de demonstrar resolutividade frente às necessidades de saúde das pessoas, bem como vínculos e responsabilizações. Há dificuldades de acesso e a prática é marcada por frieza e pouca participação. Isto porque os resquícios históricos do modelo biomédico ainda movimentam práticas individuais e impessoais, ao contrário de centralizar-se no usuário e no trabalho em equipe; o Sistema desenvolve-se burocratizado e verticalizado, desrespeitando, não raramente, o direito dos usuários ao cuidado integral. Além disto, influi também o desconhecimento do próprio funcionamento do SUS por parte de usuários e trabalhadores.

No desenvolvimento do presente relato, optou-se por informar ao leitor como o serviço era oferecido e, posteriormente, que mudanças foram implementadas.

\section{DESENVOLVIMENTO DA PROPOSTA DE ACOLHIMENTO: O ANTES E O DEPOIS}

A necessidade de implantação do acolhimento se deu primeiramente por meio da observação feita pela enfermeira da equipe sobre o modo como estava organizado o processo de trabalho e 
como eram realizados os atendimentos na UBS. O exercício cotidiano de observação despertou a importância de iluminar, na prática, as premissas da Atenção Básica do SUS, principalmente o seu caráter preferencial de porta de entrada aos serviços de saúde, e a necessidade de trabalho em equipe em território delimitado.

Em seu dia a dia, a enfermeira vinha tomando ciência de uma realidade desoladora: a fila de pessoas começava a se formar na frente da UBS às quatro horas da manhã, apesar de a Unidade abrir as portas às sete horas da manhã e fechar às sete horas da noite. Instigada pela necessidade de transformação deste cenário, ela resolveu dar voz às pessoas. Ao questioná-las sobre o porquê levantavam tão cedo para se dirigir à Unidade, as pessoas responderam que este era o caminho para tentar atendimento, uma vez que já haviam saído da Unidade com respostas negativas, tendo que retornar no outro dia mais cedo ainda. Isto acontecia pelo fato de que diariamente eram liberadas fichas de porta para atendimento médico. Até então, um cartaz era colocado pela recepcionista na porta de entrada da UBS, no final do expediente de trabalho, com o número de consultas disponibilizadas para o dia posterior. Observava-se que esse modus operandi era uma maneira de "expulsar" os usuários, uma vez que estes, ao visualizarem a fila e o número de fichas anexadas no cartaz, acabavam por ir embora. $\mathrm{O}$ fato era que havia uma demanda reprimida que não chegava a ser conhecida. Reforçava-se a lógica médico-centrada, pois a população - e, por vezes, a equipe - não vislumbrava a possibilidade de atendimento por outro profissional que não o médico. Em outra abordagem, quando as fichas de porta terminavam, não era possível a identificação dos motivos pelos quais as pessoas procuravam o serviço de saúde.

As ações de Atenção Básica representam o comando do fluxo no SUS. Esta condição aumenta, ainda mais, a necessidade de, para citar Souza e Lopes (2003), os seus serviços encontrarem formas de acolher os usuários, diferente daquela em que se passam horas e horas em uma fila para, ao final, receber a informação de que não há mais disponibilidade de consultas e/ou fichas de atendimento.

Em interlocução com o problema da fila, surgiu outra questão, do âmbito da programação das agendas dos profissionais médico e enfermeiro: considerando que essas agendas estavam organizadas para atender grupos específicos, no período vespertino, tais como pessoas com hipertensão e/ou com diabetes, gestantes (pré-natal), crianças (puericultura); considerando que eram liberadas fichas de porta para qualquer tipo de atendimento clínico, no período matutino, no caso de uma pessoa, por exemplo, gestante ou portadora de hipertensão vir para a fila da UBS às quatro da manhã, além de ela enfrentar a fila, ela não teria a garantia de agendamento de ação programática para o período vespertino.

Essa constatação contraria o que preconiza o Ministério da Saúde, em sua Portaria $N^{\circ}$ 2488 de 2011, a qual orienta que o trabalho na Atenção Básica (AB) seja realizado conforme um programa de atividades que siga a agenda de trabalho compartilhado de todos os profissionais, evitando a fragmentação do trabalho segundo critérios de problemas de saúde, ciclos de vida, sexo e patologias. Um dos focos deve ser o de prover atenção integral, contínua e organizada à população (BRASIL, 2011).

Em terceiro e, último lugar, a enfermeira percebeu que os profissionais agiam de forma diferente, diante de um mesmo problema identificado e, muitas vezes, as informações dadas aos usuários e/ou os atendimentos prestados também não seguiam um padrão, diante dos mesmos encaminhamentos. O serviço e, consequentemente, a equipe não possuíam fluxos e protocolos de atendimento, o que acabava incorrendo em encaminhamentos desnecessários, informações incorretas, dificuldades no trabalho em equipe e, consequentemente, insatisfação por parte da população. 
Werneck, Faria e Campos (2009) afirmam que a ausência de uma padronização nas ações significa fragilidade da gestão, podendo levar a uma grande variação nos modos de fazer. Em relação aos fluxos e aos protocolos de atendimento, Jacques e Gonçalo (2007, p.111) apontam para a importância que os protocolos têm na AB. Eles propiciam "processos ricos em aprendizagem organizacional e, como prescrevem racionalmente os melhores recursos a serem utilizados, são a garantia da maior probabilidade de resultados assistenciais almejados". Os autores ainda ressaltam que os mesmos são referências para a organização das diversas ações da(s) equipe(s) em uma UBS. Exigem que a equipe realize a discussão sobre a forma como trabalha. Exigem também que a equipe se organize para diagnosticar situações e problemas, planejar, decidir pela implementação das ações e por sua avaliação. E, também, possibilitam acordos, divisões de tarefas, de responsabilidades, monitoramento e avaliação.

Os problemas identificados pela enfermeira foram apresentados em uma reunião semanal da equipe. Uma agente comunitária de saúde (ACS) fez os seguintes questionamentos: a) Será que somente esses fatores, como construção de fluxos e protocolos de atendimento, mudança na agenda dos profissionais, extinção das fichas de porta com a consequente ampliação do acesso, são suficientes para garantir um acolhimento com escuta qualificada e vínculo? b) Em caso de resposta afirmativa, a escuta qualificada e o vínculo estariam presentes de forma transversal, durante todos os encontros e ações cuidadoras? c) Todos os profissionais que trabalham na Unidade compreendem as bases do processo de acolhimento? d) Seria possível corresponsabilizar a gestão central com vistas ao apoio operacional? e) Os usuários, sujeitos das ações, poderiam ser incluídos na construção da proposta?

Segundo Ceccim (2005), para produzir mudanças de práticas de atenção em saúde, é fundamental dialogar com as práticas e as concepções vigentes, problematizá-las, não no abstrato, mas no concreto do trabalho de cada equipe e assim construir novos pactos de convivência e práticas que aproximem o SUS da atenção integral à saúde. Não bastam novas informações ou regras, mesmo que precisamente bem comunicadas, senão para a mudança, transformação ou crescimento.

Nessa perspectiva, realizou-se coletivamente um planejamento em reuniões de equipe, priorizando a educação permanente que, segundo Luz (2010), tem a capacidade de facilitar o envolvimento de todos os profissionais e proporcionar a troca de saberes e a reflexão das práticas de serviço; consequentemente, possibilita uma maior interação da equipe e uma melhoria no processo de trabalho. Carotta, Kawamura e Salazar (2009, p. 48) concordam com a afirmação de Luz (2010) quando conceituam:

A educação permanente trabalha com ferramentas que buscam a reflexão crítica sobre a prática cotidiana dos serviços, sendo, por si só, um processo educativo aplicado ao trabalho que possibilita mudanças nas relações, nos processos, nos atos de saúde e nas pessoas.

Nas reuniões de equipe, foram amplamente discutidos os princípios do SUS, os conceitos de saúde e doença e as diretrizes da atenção básica (ordenação da rede, participação da comunidade, primeiro contato, acessibilidade, longitudinalidade, integralidade e coordenação do cuidado). Discutiram-se, também, alguns sentidos de acolhimento, descritos pela literatura, para que seu entendimento não ficasse restrito ao sentido coloquial atribuído à palavra, como o simplesmente cordial "receber bem", mas que incorporasse todo o processo de trabalho em saúde. Elegeu-se a compreensão de Merhy (1997) e de Franco (1999).

Para Merhy (1997, p. 71): 
O acolhimento significa a humanização do atendimento, o que pressupõe a garantia de acesso a todas as pessoas (acessibilidade universal). Diz respeito, ainda, à escuta de problemas de saúde do usuário, de forma qualificada, dando-lhe sempre uma resposta positiva e responsabilizando com a resolução do problema. Por consequência, o acolhimento deve garantir a resolubilidade que é o objetivo final do trabalho em saúde, resolver efetivamente o problema do usuário. A responsabilização para com o problema de saúde vai além do atendimento propriamente dito, diz respeito também ao vínculo necessário entre o serviço e a população usuária.

Segundo Franco (1999, p. 347), o acolhimento como diretriz operacional propõe a inversão da lógica de organização e funcionamento dos serviços de saúde, partindo de alguns princípios:

1) Atender a todas as pessoas que procuram os serviços de saúde, garantindo a acessibilidade universal. Assim, o serviço de saúde assume sua função precípua, a de acolher, escutar e dar uma resposta positiva, capaz de resolver os problemas de saúde da população.

2) Reorganizar o processo de trabalho, a fim de que este desloque seu eixo central do médico para uma equipe multiprofissional - equipe de acolhimento - que se encarrega da escuta do usuário, comprometendo-se a resolver seu problema de saúde.

3) Qualificar a relação trabalhador-usuário, que deve dar-se por parâmetros humanitários, de solidariedade e cidadania.

Em seguida, foram realizados convites para os demais profissionais que não faziam parte da equipe, como recepcionistas e auxiliares de serviços gerais. A participação desses trabalhadores era importante, já que se buscava desenvolver a ideia do acolhimento como postura desenvolvida por todos os profissionais, procurando um maior vínculo com a comunidade. Merhy, Cecílio e Nogueira Filho (1991) afirmam que a recepção é um ponto estratégico no atendimento de uma Unidade de Saúde, e por isso deve contar com um profissional qualificado que possa fornecer informações e encaminhamentos corretos. Convites feitos, convites aceitos.

A partir de então, as reuniões contaram com a presença de membros da comunidade. $\mathrm{O}$ objetivo foi conhecer as percepções deles a respeito da introdução de agendamento e acolhimento, em substituição às fichas de portas. Nos encontros, debateu-se sobre alguns fundamentos da Estratégia Saúde da Família. Trabalhadores e usuários desenharam a proposta.

Subsequentemente, agendou-se e realizou-se uma reunião com as coordenadoras de ESF e com a gestão da Secretaria Municipal de Saúde (SMS). Na ocasião, a proposta de implantação do acolhimento foi apresentada com a intenção desta ser um projeto para os demais serviços de saúde do município.

Após o término desse processo, que durou nove meses, cujos trabalhadores, usuários e gestores estavam sensibilizados com a proposta de acolhimento, iniciou-se a operacionalização do aprendizado coletivo: construiu-se um fluxograma com o objetivo de nortear as ações de acolhimento na Unidade (Figura 1).

Realizou-se, também, uma escala dos profissionais da equipe que deveriam estar sempre na Unidade para acolher os usuários, incluindo todos os membros da equipe. Alteraram-se as agendas dos profissionais, no âmbito da organização por horários, sem fragmentar em especificidades por ciclos de vida. Esta agenda foi qualificada como "agenda integrada”, já que a mesma possibilitaria 
qualquer tipo de atendimento de acordo com a necessidade dos usuários. As consultas passaram a ser agendadas em qualquer dia e horário de funcionamento da UBS, até mesmo por telefone.

Essas mudanças não somente ampliaram o acesso dos usuários ao serviço, como aumentaram o vínculo entre eles e a equipe. Por consequência, a iniciativa foi ao encontro da satisfação da população, além de aumentar o comprometimento da equipe sobre o trabalho realizado, no que se refere à responsabilização e dedicação.

\section{FLUXOGRAMA DE ATENDIMENTO}

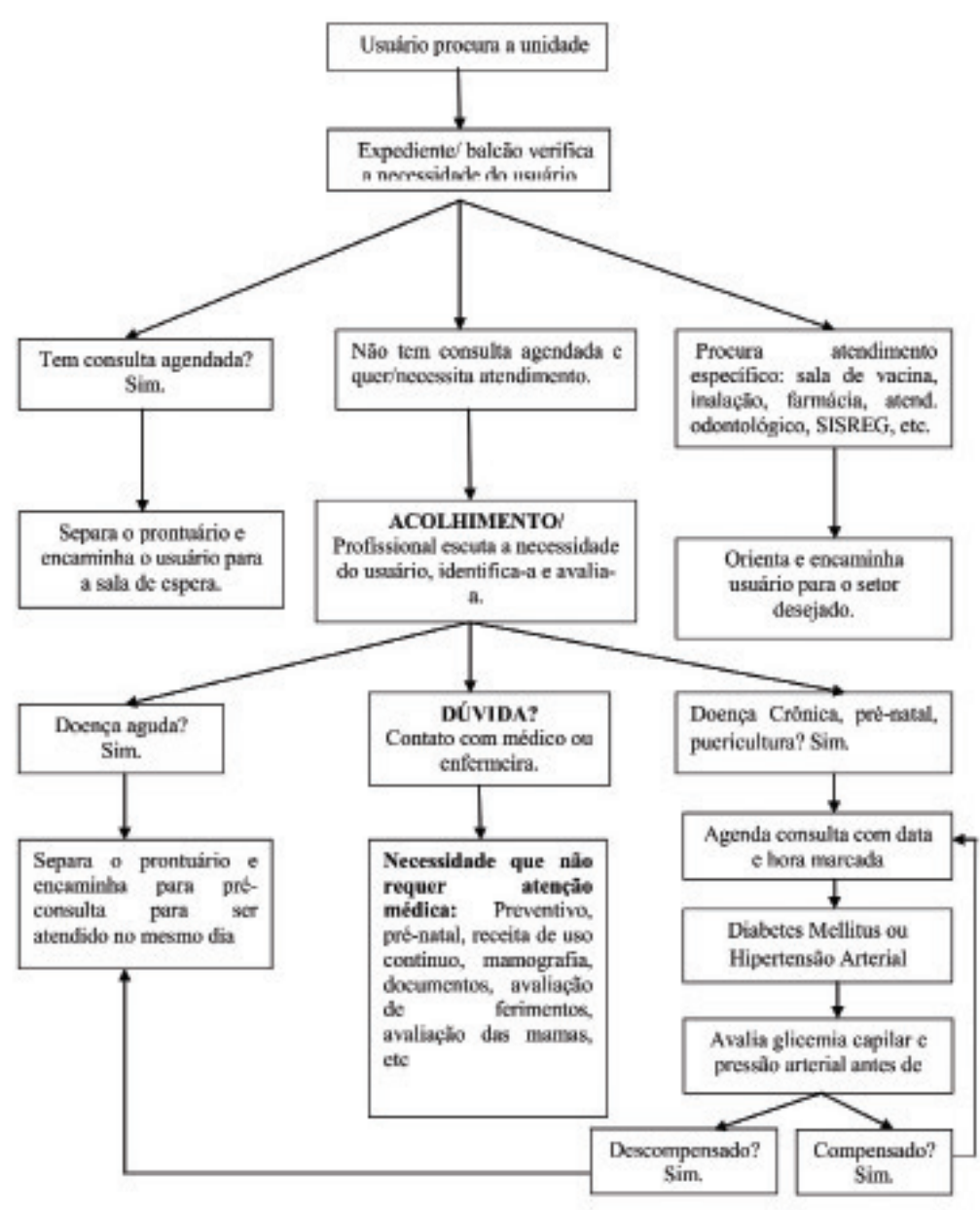

Figura 1:Fluxogramadaptado Caderno $A B n^{2} 28 \mathrm{MS}$.

CONSIDERAÇÕES FINAIS

Apresenta-se um relato de experiência sobre uma proposta de acolhimento, desenvolvida por uma equipe de Saúde da Família de uma UBS de um município do Sul do Brasil. A proposta gerou um novo fluxo de acesso às ações e aos serviços e uma nova organização do processo de trabalho da equipe, a partir da extinção de fichas de porta e da produção de uma agenda integrada.

Motivada pelo dispositivo educação permanente, a iniciativa possibilitou transformações na equipe, no sentido de praticar "outro jeito" de receber as pessoas da comunidade que se dirigem à UBS em busca de alívio para suas dores. Este "outro jeito" qualificou o atendimento das pessoas que residem no território da referida UBS, bem como o entendimento da população e dos profissionais sobre as diretrizes da $\mathrm{AB}$ e imagem-objetivo de acolhimento. 
Foi possível perceber que a qualificação do acesso às ações e aos serviços da atenção básica requer disposição dos atores envolvidos, "vontade política para” garantir que o sofrimento, tanto inespecífico como específico, seja acolhido com base no reconhecimento na dignidade da pessoa humana. A grandeza do acolhimento está exatamente na simplicidade de sua complexidade.

No entanto, apesar de todo o impacto positivo observado na UBS, permanece o desafio de se ampliar o olhar para além da assistência médica e, nesse sentido, tornam-se necessárias mudanças não somente nos processos de trabalho, mas, também, de gestão e de formação a partir de uma ótica usuário-centrado.

\section{REFERÊNCIAS}

BRASIL. Ministério da Saúde. Secretaria de Atenção à Saúde. Núcleo Técnico da Política Nacional de Humanização. Acolhimento nas Práticas de Produção de Saúde. Série B. Textos Básicos de Saúde, Brasília; 2.ed.; 2010a.

BRASIL. Ministério da Saúde. Secretaria de Atenção à Saúde. Departamento de Atenção Básica. Acolhimento à demanda espontânea. Série A. Normas e Manuais Técnicos. Cadernos de Atenção Básica, Brasília, v. 1, n. 28, 2013.

BRASIL. Ministério da Saúde. HumanizaSUS. Brasília: Ministério da Saúde, 2010b. Disponível em: <http://vsms.saude.gov.br/HTML/PT/dicas/167acolhimento.html>. Acesso em: 16 out. 2016.

BRASIL. Ministério da Saúde. Portaria no 2.488, de 21 de outubro de 2011. Aprova a Política Nacional de Atenção Básica, estabelecendo a revisão de diretrizes e normas para a organização da Atenção Básica, para a Estratégia Saúde da Família (ESF) e o Programa de Agentes Comunitários de Saúde (PACS). Disponível em: <http://bvsms.saude.gov.br/bvs/ saudelegis/gm/2011/prt2488_21_10_2011.html>. Acesso em: 26 nov. 2017.

CARMAGNANI, M. I. S. Grupo de Trabalho de Humanização do HSP/UNIFESP. 2005. Disponível em: <http://www.unifesp.br/hsp/humaniza/index.htm>. Acesso em: 20 jul. 2017.

CAROTTA, F.; KAWAMURA, D.; SALAZAR, J. Educação em Saúde: uma estratégia de gestão para pensar, refletir e construir práticas educativas e processos de trabalho. Saúde Sociedade, São Paulo, v.18, n.1, p.48, 2009.

CECCIM, R. B. Educação permanente em saúde: desafio ambicioso e necessário. Interface Comunicação, Saúde, Educação, Porto Alegre, v.9, n.16, p.161-168, 2005.

FRANCO, T. B. et al. O acolhimento e os processos de trabalho em saúde: o caso de Betim (MG). Cadernos de Saúde Pública, Rio de Janeiro, v.15, n.2, p.345-353, 1999.

JACQUES, E. J.; GONÇALO, C. R. Gestão estratégica do conhecimento baseada na construção de protocolos médico-assistenciais: o compartilhamento de ideias entre parcerias estratégicas como vantagem competitiva. Revista de Administração e Inovação, São Paulo, v.4, n.1, p.106-124, 2007.

LYRA, M. et al. Welcoming in the primary health care of the Health Unique System: a theoretical and epistemological perspective. Fiep Bulletin, v. 87, p. 122-129, 2016.

LUZ, F. M. Educação Permanente em Saúde (EPS): Uma estratégia que possibilita transformações no processo de trabalho. Varginha: MG, 2010.

MAEYAMA, M. A. A Construção do Acolhimento: a proposta de mudança do processo de trabalho em saúde do município de Bombinhas. Secretaria Municipal de Saúde de Bombinhas: Bombinhas, 2007. 
MERHY, E. E. Em busca do tempo perdido: a micropolítica do trabalho vivo em saúde. In: MERHY, E. E.; ONOCKO, R. (Org). Agir em Saúde: um desafio para o público. São Paulo: Hucitec, 1997.

MERHY, E. E.; CECÍlio, L. C. O.; NOGUEIRA FILHO, R. C. Por um modelo tecno-assistencial da política de saúde em defesa da vida: contribuição para as conferencias de saúde. Revista Saúde em Debate, Londrina, v.33, 1991.

SOUZA, A. C.; LOPES, N. M. J. M. Acolhimento: responsabilidade de quem? Um relato de experiência. Revista Gaúcha de Enfermagem, Porto Alegre, v.24, n.1, abr. 2003.

WERNECK, M. A. F; FARIA, H. P.; CAMPOS, K. F.C. Protocolo de cuidados à saúde e de organização do serviço. Belo Horizonte: Nescon/UFMG, Coopmed, 2009. 